\title{
RF Impairments in High-rate Wireless Systems - understanding the impact of TX/RX-asymmetry
}

\author{
Tim C. W. Schenk ${ }^{\triangleright}$ and Erik R. Fledderus*o \\ $\triangleright$ Philips Research, Distributed Sensor Systems department, HTC 37, Eindhoven, The Netherlands, tim.schenk@philips.com \\ * Eindhoven University of Technology, Radio Communications Chair, PO Box 513, 5600 MB, Eindhoven, The Netherlands \\ - TNO Information and Communication Technology, Brassersplein 2, 2612 CT, Delft, The Netherlands, erik.fledderus@tno.nl
}

\begin{abstract}
Dealing with radio frequency (RF) front-end impairments will be one of the major design challenges for nextgeneration wireless communication systems due to conflicting requirements, such as high data rate, low cost and low power consumption. The use of digital compensation of the imperfections appears a very promising method to meet specifications. Pursuing that path, however, requires thorough understanding of the influence of the RF front-end non-idealities on the received signal and the resulting system performance.

To this end, this paper reviews the impact of three important impairments, namely, phase noise, IQ imbalance and nonlinearities, on the performance of next-generation high-rate wireless systems. A specific focus is on the difference between transmitter (TX) and receiver (RX) incurred imperfections. Moreover, a generalized error model to capture to aggregate influence of different impairments is presented.
\end{abstract}

\section{INTRODUCTION}

The design of new high data rate radio interfaces opens up a continuing search for solutions to increasingly conflicting requirements. Higher data rates require higher sampling rates, leading to higher energy consumption. However, the improvement of battery capacity is not keeping pace with the increasing demands. Moreover, these higher bandwidths require wireless systems to operate in the high radio frequency (RF) bands. Creating efficient and high-performance integrated circuits for these high carrier frequencies is very challenging. At the same time one can observe a clear move of wireless communications solutions into the commodity markets. Consequently, size and cost become key parameters for design and implementation.

These conflicting requirements are from all times - the design of e.g wireless networks has always turned around striking a balance between capacity, coverage, quality, and costs. Network planners know that fixing three of the design parameters results in an outcome for the remaining one. In case all parameters are fixed, the solution space may turn out to be empty. This situation is dawning for next generation high data rate radio transceivers, where competition and market pressure result in a small or empty design space.

Being on the edge of production accuracy, the impact of imperfections can be equal to not meeting the specs. A further decrease of margins will result in higher costs, so new solutions need to be found. An intuitive path, that is actually very promising, is the increase of the number of degrees of freedom. More precisely, meeting the specs is not only a matter anymore for e.g. the RF analog domain, but can be accomplished by involving digital compensation. Pursuing this path requires excellent knowledge of the occurring impairments, and the way these imperfections carry over via nonlinear processing and a radio channel into the receiver of the system [1], [2].

Since this understanding is system dependent, it is advantageous that both orthogonal frequency division multiplexing (OFDM) and multiple-input multiple-output (MIMO) are dominant in next-generation high-rate wireless standards. Examples are OFDM-based ultra-wideband solutions for personal area networks in ECMA 368, MIMO OFDM for local area networks in IEEE 802.11n, OFDM and MIMO for metropolitan area networks in IEEE 802.16e, MIMO OFDM in 3GLTE for wide area networks, and OFDM and MIMO in digital broadcasting networks. In addition, these techniques are also prime candidates for the software defined radio/cognitive radio paradigm that will dominate the $5 \mathrm{G}$-discussion.

Besides being the basis for many high data rate wireless standards, the main advantages of OFDM are its high spectral efficiency and its ability to use the multipath channel to its advantage. It has several disadvantages, however, that mainly lie in the constraints it puts on the quality of the analog RF front-end of both transmitter (TX) and receiver (RX). Hence, for reliable communication a high linearity, a highly stable RF oscillator, and high matching between the in-phase (I) and quadrature-phase $(\mathrm{Q})$ branches is required. These are the imperfections considered in the rest of this paper.

Finally, it has been shown that the influence of imperfections at TX and RX is quite different in fading channel environments, see e.g. [3]. In the downlink a (cheap, lowpower) user terminal acts as RX and a (more expensive) base station/access point as TX and vice versa in the uplink. The imperfections will likely be dominant at the terminal side of the link, while processing power will be mostly available at the base station side of the link. New architectures, such as ad-hoc and mesh networks, will, however, change this picture. Hence, it is relevant to review the impact of $\mathrm{TX} / \mathrm{RX}$ impairment asymmetry, as will be done in this paper.

The outline of this paper is as follows. First the MIMO OFDM system model is treated in Section II. Subsequently, Sections III to $\mathrm{V}$ review the performance impact of the different RF imperfections. A generalized error model is then introduced in Section VI. Finally, the paper ends with conclusions and a discussion in Section VII. 


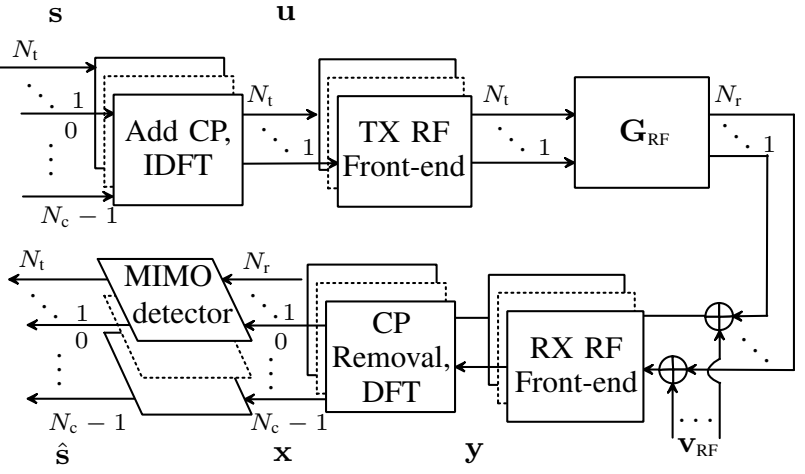

Fig. 1. Block diagram for a multiple-antenna OFDM system.

\section{SYSTEM MODEL}

Consider a MIMO OFDM system with $N_{\mathrm{t}}$ TX and $N_{\mathrm{r}} \mathrm{RX}$ antennas, referred to in the following as a $N_{\mathrm{t}} \times N_{\mathrm{r}}$ system, applying $N_{\mathrm{c}}$ subcarriers of which $2 K$ carry data symbols. A schematic diagram of such a system is depicted in Fig. 1.

Let us define the $m$ th $N_{\mathrm{c}} N_{\mathrm{t}} \times 1$ MIMO OFDM vector to be transmitted as $\mathbf{s}_{m}=\left[\mathbf{s}_{m, 0}^{T}, \ldots, \mathbf{s}_{m, N_{\mathrm{c}}-1}^{T}\right]^{T}$, where $\mathbf{s}_{m, k}$ denotes the $N_{\mathrm{t}} \times 1$ frequency domain MIMO transmit vector for the $k$ th subcarrier. The $n_{\mathrm{t}}$ th element of the subvector $\mathbf{s}_{m, k}$ corresponds to the $n_{\mathrm{t}}$ th TX branch. In the TX digital baseband, $\mathbf{s}_{m}$ is transformed to $\mathbf{u}_{m}$ by the use of the inverse discrete Fourier transform (IDFT) and the addition of a cyclic prefix (CP) of $N_{\mathrm{g}}$ samples. We assume that by design $N_{\mathrm{g}}$ is at least equal to the channel impulse response length, preventing intersymbol interference. The baseband TX vector $\mathbf{u}_{m}$ is input to the $N_{\mathrm{t}}$ TX RF front-ends, which up-convert the signals to be transmitted through the wireless channel, denoted by $\mathbf{G}_{\mathrm{RF}}$.

At the RX, the signals obtained at the $N_{\mathrm{r}}$ antennas are down-converted using the $N_{\mathrm{r}} \mathrm{RX}$ RF front-ends, yielding the $m$ th received $\left(N_{\mathrm{g}}+N_{\mathrm{c}}\right) N_{\mathrm{r}} \times 1$ complex baseband vector $\mathbf{y}_{m}$. The receiver noise is here denoted as $\mathbf{v}_{\mathrm{RF}}$. In the $\mathrm{RX}$ digital baseband the $\mathrm{CP}$ is removed and the discrete Fourier transform (DFT) is applied, which yields the $N_{\mathrm{c}} N_{\mathrm{r}} \times 1$ vector $\mathbf{x}_{m}$. For perfect timing and frequency synchronization, and no frontend imperfections, $\mathbf{x}_{m}$ can be written as [4, Chapter 2]

$$
\mathbf{x}_{m}=\mathbf{H} \mathbf{s}_{m}+\mathbf{n}_{m}
$$

where $\mathbf{n}_{m}$ is the frequency domain baseband version of the additive white Gaussian RX noise (AWGN) $\mathbf{v}_{\mathrm{RF}}$. The $N_{\mathrm{r}} N_{\mathrm{c}} \times N_{\mathrm{t}} N_{\mathrm{c}}$ block diagonal frequency-domain channel matrix $\mathbf{H}$ is given by $\operatorname{diag}\left(\mathbf{H}_{0}, \ldots, \mathbf{H}_{N_{\mathrm{c}}-1}\right)$, where $\mathbf{H}_{k}$ denotes the $N_{\mathrm{r}} \times N_{\mathrm{t}}$ baseband-equivalent MIMO channel matrix for the $k$ th subcarrier. Note that we omitted the index $m$ for the channel matrix, since we assume a quasi-static channel in this paper.

Due to the block-diagonal property of the channel matrix, the received signal vector for the $k$ th subcarrier can be written as $\mathbf{x}_{m, k}=\mathbf{H}_{k} \mathbf{s}_{m, k}+\mathbf{n}_{m, k}$. Subsequently, MIMO detection can be applied per subcarrier to retrieve an estimate of the TX signal. In this paper we will restrict our analyses to space division multiplexing (SDM) systems, where independent data streams are transmitted from the TX branches, although results can easily be generalized. Also, for simplicity, we only consider zero-forcing (ZF) MIMO processing, where the estimate of the TX signal is found by multiplying $\mathbf{x}_{m, k}$ with the inverse of a channel estimate $\hat{\mathbf{H}}_{k}$, yielding

$$
\hat{\mathbf{s}}_{m, k}=\hat{\mathbf{H}}_{k}^{\dagger} \mathbf{x}_{m, k}=\mathbf{s}_{m, k}+\boldsymbol{\varepsilon}_{m, k},
$$

where ${ }^{\dagger}$ denotes the pseudo-inverse. For perfect channel knowledge and only AWGN, the error $\boldsymbol{\varepsilon}_{m, k}$ equals $\mathbf{H}_{k}^{\dagger} \mathbf{n}_{m, k}$.

\section{Phase NoISE}

Let us first consider the performance impact of the nonidealities of the RF reference signal. Since, in general, the disturbance of the amplitude of the oscillator output is marginal, most influence of imperfections is noticeable in a random deviation of its output frequency. These frequency deviations are often modeled as a random excess phase, and referred to as phase noise (PN).

\section{A. System Model}

Under the influence of both TX and RX PN, the system model in (1) is transformed into [3]

$$
\mathbf{x}_{m}=\left(\mathcal{G}_{\mathrm{r}, m} \otimes \mathbf{I}_{N_{\mathrm{r}}}\right) \mathbf{H}\left(\mathcal{G}_{\mathrm{t}, m} \otimes \mathbf{I}_{N_{\mathrm{t}}}\right) \mathbf{s}_{m}+\mathbf{n}_{m},
$$

where $\otimes$ and $\mathbf{I}_{M}$ denote the direct matrix product and the $M$ dimensional identity matrix, respectively. Here $\mathcal{G}_{\mathrm{t}, m}$ and $\mathcal{G}_{\mathrm{r}, m}$ are Toeplitz matrices, modeling the influence of TX and RX $\mathrm{PN}$, respectively. The $(k, l)$ th element of $\mathcal{G}_{\mathrm{x}, m}$ is given by

$$
\gamma_{k-l, m}^{\mathrm{x}}=\left(1 / N_{\mathrm{c}}\right) \sum_{i=0}^{N_{\mathrm{c}}-1} e^{j \theta_{\mathrm{x}, m}\left(N_{\mathrm{g}}+i\right)} e^{-j \frac{2 \pi\{k-l\} i}{N_{\mathrm{c}}}} .
$$

where $\mathrm{x} \in\{\mathrm{t}, \mathrm{r}\}$ and $\theta_{\mathrm{x}, m}(i)$ denotes the PN during the $i$ th sample of the $m$ th OFDM symbol. Note that for a system not experiencing PN, we have that $\gamma_{0, m}^{\mathrm{x}}=1$ and $\gamma_{k-l, m}^{\mathrm{x}}=0$ for $k \neq l$. Consequently, $\mathcal{G}_{\mathrm{t}}$ and $\mathcal{G}_{\mathrm{r}}$ reduce to identity matrices and all carriers are orthogonal.

All elements on the diagonal of $\mathcal{G}_{\mathrm{t}}$ and $\mathcal{G}_{\mathrm{r}}$ are equal, i.e., $\gamma_{0}^{\mathrm{t}}$ and $\gamma_{0}^{\mathrm{r}}$, respectively, and have approximately unity amplitude for moderate PN. These diagonal elements cause a rotation of the wanted signals and as this rotation is equal for all carriers, it is often called the common phase error (CPE). The other elements in $\mathcal{G}_{\mathrm{x}}$ cause inter-carrier interference (ICI). We use this to rewrite (3) to

$$
\mathbf{x}_{m}=\gamma_{0, m} \mathbf{H} \mathbf{s}_{m}+\boldsymbol{\xi}_{m}+\mathbf{n}_{m}
$$

where $\gamma_{0, m}=\gamma_{0, m}^{\mathrm{r}} \gamma_{0, m}^{\mathrm{t}}$ and $\boldsymbol{\xi}_{m}$ represents the ICI contribution for the $m$ th symbol and can be written as

$$
\begin{aligned}
\boldsymbol{\xi}_{m}= & \left(\boldsymbol{\varphi}_{\mathrm{r}, m} \otimes \mathbf{I}_{N_{\mathrm{r}}}\right) \mathbf{H}\left(\boldsymbol{\varphi}_{\mathrm{t}, m} \otimes \mathbf{I}_{N_{\mathrm{t}}}\right) \mathbf{s}_{m} \\
& +\gamma_{0, m}^{\mathrm{r}} \mathbf{H}\left(\boldsymbol{\varphi}_{\mathrm{t}, m} \otimes \mathbf{I}_{N_{\mathrm{t}}}\right) \mathbf{s}_{m}+\gamma_{0, m}^{\mathrm{t}}\left(\boldsymbol{\varphi}_{\mathrm{r}, m} \otimes \mathbf{I}_{N_{\mathrm{r}}}\right) \mathbf{H} \mathbf{s}_{m} .(6)
\end{aligned}
$$

Here $\boldsymbol{\varphi}_{\mathrm{t}, m}=\mathcal{G}_{\mathrm{t}, m}-\gamma_{0, m}^{\mathrm{t}} \mathbf{I}_{N_{\mathrm{t}} N_{\mathrm{c}}}$ and $\boldsymbol{\varphi}_{\mathrm{r}, m}$ has the same structure as $\varphi_{\mathrm{t}, m}$. Thus, the first term in (5) is the desired signal times some common rotation $\gamma_{0, m}$. The second term models the ICI, as worked out in (6). It is important to note that this term contains the complex channel matrix and will thus exhibit properties of the channel. The last term in (5) is the AWGN.

The average amplitude of the elements of $\mathcal{G}_{\mathrm{x}, m}$ is dependent on the power spectral density (PSD) of the PN of the applied oscillator. In general one can say that low frequencies in the 
PSD contribute to the amplitude of elements $\gamma_{q, m}^{\mathrm{x}}$ with low $q$, and high frequency content contributes to the elements with high $q$. More specifically, frequency components $<\left|f_{\mathrm{s}} / N_{\mathrm{c}}\right|$ in the PN PSD contribute to the CPE and frequency components $>\left|f_{\mathrm{s}} / N_{\mathrm{c}}\right|$ to the ICI, as previously shown in [5, Chapter 5].

The influence of the CPE is generally the most severe, since it can arbitrarily rotate the complex constellation, e.g. $M$-QAM or $M$-PSK, used for modulation of the subcarriers, resulting in block errors in detection. An obvious solution would be the use of differential modulation and detection. This can, however, result in a considerable degradation in achievable rate. That is why often a phase tracking algorithm is applied, either by using a PLL in the frequency synthesizer or by applying of a digital CPE correction algorithm. It has been shown that these algorithms can, with reasonable complexity, fully suppress the influence of the phase shifts due to the CPE, see e.g. [6]. Currently, such CPE compensation solutions are being commonly applied in OFDM-based wireless solutions.

\section{B. ICI distribution}

After CPE compensation, the additive ICI term is the main performance limiting factor in $\mathrm{PN}$-impaired systems. Therefore we next review the performance impact of the ICI. The ICI term has been previously studied in several contributions in literature, see e.g. [6]-[8]. Many of these papers, implicity of explicitly, assume that the ICI term has a complex Gaussian distribution due to the central limit theorem. It has been indicated in [9], [10] and proven in [11], however, that the Gaussian approximation is not valid. In [11] it is shown that the ICI distribution has heavier tails than the normal distribution. Since the tails of the distribution determine the bit-error rate (BER) performance, the use of the Gaussian approximation of the ICI results in an underestimation of the impact of $\mathrm{PN}$ on system performance. Therefore a correct limit distribution for the ICI term was derived in [11], under the assumption of a large number of subcarriers. It was shown numerically, however, that the derived expression already holds for a modest number of subcarriers.

The validity of this limit distribution is illustrated in Fig. 2, which depicts results from BER simulations for a system experiencing PN and no AWGN. Results are given for an IEEE 802.11a-like system experiencing Brownian PN as function of the $-3 \mathrm{~dB}$ PN bandwidth $\beta$ for different modulations. These results can be interpreted as the high SNR flooring of the BER curve for a certain $\beta$, i.e., the maximum achievable BER performance for a certain level of PN. These results are depicted together with results from simulations where the ICI is modeled as a zero-mean complex Gaussian noise source at the RX, i.e., the commonly used approach in previous literature, where the variance of the noise equals that of the actual ICI. Finally, results are included from simulations where the influence of the ICI is modeled using the limit distribution.

It can be concluded from Fig. 2 that for both modulation formats the Gaussian approximation of the ICI only provides reliable BER results for very high values of $\beta$, where the BER is so high that reliable data transfer is almost not possible.

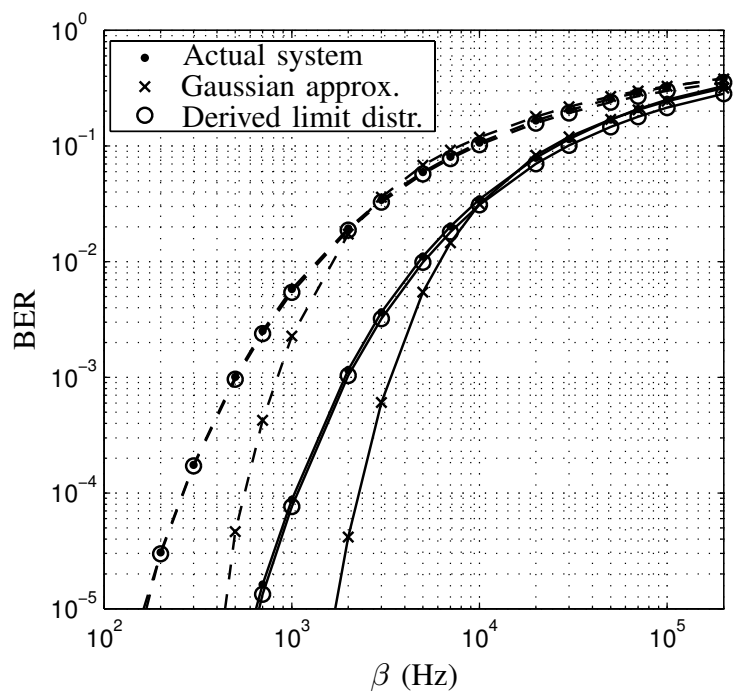

Fig. 2. BER results for a system applying 16-QAM (in solid lines) and 64-QAM (in dashed lines) modulation. Results are included for a system experiencing Brownian PN and applying perfect CPE correction (Actual system), a system modelling the resulting ICI as an equivalent complex Gaussian process (Gaussian approx.) and a system modeling the resulting ICI according to the derived limit distribution (Derived limit distr.).

This confirms findings in [9] and [10], where simulations were used to show that the Gaussian model only holds for very high values of $\beta$. The BER found from simulations with the approximate expression for our limit distribution from [11], on the other hand, shows good agreement with the BER found from simulations with the actual $\mathrm{PN}$-impaired system.

\section{ICI-related error term}

One can conclude from (5) that the impact of TX and RX $\mathrm{PN}$ on the CPE is equal. In contrast, the structure of (6) reveals specific differences between the TX and RX PN impact due to the ICI. To clarify this difference, we will study the error term after ZF-based MIMO processing, see (2), for a system experiencing PN and applying perfect CPE correction. For convenience we omit the influence of AWGN. The error term is then given by $\boldsymbol{\varepsilon}_{m, k}=\mathbf{H}_{k}^{\dagger} \boldsymbol{\xi}_{m, k}$, which equals $\boldsymbol{\varepsilon}_{\mathrm{t}, m, k}=$ $\sum_{l=0, l \neq k}^{N_{\mathrm{c}}-1} \gamma_{k-l}^{\mathrm{TX}} \mathbf{s}_{m, l}$ and $\varepsilon_{\mathrm{r}, m, k}=\mathbf{H}_{k}^{\dagger} \sum_{l=0, l \neq k}^{N_{\mathrm{c}}-1} \gamma_{k-l}^{\mathrm{RX}} \mathbf{H}_{l} \mathbf{s}_{m, l}$ for TX and RX PN, respectively. Note that the expression for RX PN does, in contrast to that for TX PN, contain the channel influence.

It is not difficult to see that for a frequency flat channel, i.e., $\mathbf{H}_{k}=\mathbf{H}_{l}$, the expression for the error term is equal for TX and RX PN noise. Hence, there is no difference between the impact of TX and RX PN for this special case. For the earlier considered Brownian PN model, the average power of the elements of the error vector is given by [3]

$P_{\mathrm{t}}=P_{\mathrm{r}, \text { flat }}=2 \sigma_{\mathrm{s}}^{2} \pi \beta T_{\mathrm{s}}\left(N_{\mathrm{c}}^{2}-1\right) /\left(3 N_{\mathrm{c}}\right) \approx 2 \sigma_{\mathrm{s}}^{2} \pi \beta T_{\mathrm{s}} N_{\mathrm{c}} / 3$,

for $N_{\mathrm{c}}$ equal to a power of 2 and where $T_{\mathrm{s}}$ and $\sigma_{\mathrm{s}}^{2}$ are the sampling time and signal power, respectively.

In a similar way one can derive a result for the special case of per subcarrier i.i.d. Rayleigh fading and $N_{\mathrm{r}}>N_{\mathrm{t}}$ for RX PN, yielding $P_{\mathrm{r}, \text { i.i.d. }} \approx P_{\mathrm{t}} N_{\mathrm{t}} /\left(N_{\mathrm{r}}-N_{\mathrm{t}}\right)$. These analytical results have been confirmed by simulations results presented in [3]. 
Overall we can conclude that the power of the error term for RX PN is equal to that of the TX case for a flat Rayleighfaded channel. For i.i.d. Rayleigh-faded channels, however, the RX PN is concluded to have less impact than TX PN when $N_{\mathrm{r}}>2 N_{\mathrm{t}}$ and more impact when $N_{\mathrm{t}}<N_{\mathrm{r}}<2 N_{\mathrm{t}}$.

The observed differences between the influence of TX and RX PN in systems experiencing fading channels can intuitively be understood as follows. The ICI term effectively creates an extra TX or RX additive noise source for the TX and RX PN, respectively. Since the TX noise source is located in front of the channel, it will result in high SNR flooring in the BER curves according to the AWGN channel BER performance, i.e., independent of the MIMO configuration. Therefore, also the power of the resulting error term is independent of the dimensions of the channel matrix. For RX PN, in contrast, the ICI source is behind the fading channel. As such, its influence is similar to that of the commonly studied AWGN, i.e., the BER performance (and thus the BER flooring) depends on the MIMO configuration. Consequently, the power of the resulting detection error depends on the MIMO dimensions, i.e., the number of TX and RX branches. For the special case of flat fading, the ZF MIMO detection removes the channel influence in the RX PN caused ICI term. As such, its influence is effectively equal to that of a TX noise source.

\section{IQ IMBALANCE}

Another important RF front-end non-ideality is imperfect matching between the I and Q branch, which will result in a phase and an amplitude imbalance and is often referred to as IQ imbalance. Although IQ imbalance can occur in different transceiver architectures, its influence is very pronounced in the popular direct-conversion architecture [12]. Therefore this section reviews the influence of IQ imbalance on the performance of a direct conversion-based MIMO OFDM system.

\section{A. System Model}

If we for notational convenience renumber the subcarriers, the received MIMO signal vector for the $k$ th data-conveying subcarrier can be written as [13]:

$$
\begin{aligned}
\mathbf{x}_{m, k}= & \left(\mathbf{K}_{1} \mathbf{H}_{k} \mathbf{G}_{1}+\mathbf{K}_{2} \mathbf{H}_{-k}^{*} \mathbf{G}_{2}\right) \mathbf{s}_{m, k}+\mathbf{K}_{1} \mathbf{n}_{m, k} \\
& +\left(\mathbf{K}_{2} \mathbf{H}_{-k}^{*} \mathbf{G}_{1}^{*}+\mathbf{K}_{1} \mathbf{H}_{k} \mathbf{G}_{2}^{*}\right) \mathbf{s}_{m,-k}^{*}+\mathbf{K}_{2} \mathbf{n}_{m,-k}^{*},
\end{aligned}
$$

for $k \in\{-K, \ldots,-1,1, \ldots, K\}$. Here the diagonal imbalance matrices are given by $\mathbf{G}_{1}=\left(\mathbf{I}+\boldsymbol{g}_{\mathrm{t}} e^{j \boldsymbol{\phi}_{\mathrm{t}}}\right) / 2$, $\mathbf{G}_{2}=\mathbf{I}-\mathbf{G}_{1}^{*}, \quad \mathbf{K}_{1}=\left(\mathbf{I}+\boldsymbol{g}_{\mathrm{r}} e^{-j \phi_{\mathrm{r}}}\right) / 2 \quad$ and $\quad \mathbf{K}_{2}=\mathbf{I}-\mathbf{K}_{1}^{*}$. In (8) the phase and amplitude imbalance are modeled by the matrices $\phi_{\mathrm{x}}=\operatorname{diag}\left\{\phi_{\mathrm{x}, 1}, \phi_{\mathrm{x}, 2}, \ldots, \phi_{\mathrm{x}, N_{\mathrm{x}}}\right\}$ and $\boldsymbol{g}_{\mathrm{x}}=\operatorname{diag}\left\{g_{\mathrm{x}, 1}, g_{\mathrm{x}, 2}, \ldots, g_{\mathrm{x}, N_{\mathrm{x}}}\right\}$, respectively, with $\mathrm{x} \in\{\mathrm{t}, \mathrm{r}\}$. We note that $\phi_{\mathrm{x}}$ and $\boldsymbol{g}_{\mathrm{x}}$ contain different imbalance values for the different TX/RX streams, since different TX/RX branches will likely exhibit unequal mismatches. For allowing frequency selective IQ imbalance, due to e.g. filter mismatch, also $\mathbf{G}$ and $\mathbf{K}$ are considered to be a function of $k$.

It can be concluded from (8) that the $m$ th received frequency-domain vector on the $k$ th subcarrier is given by the TX vector on that carrier, i.e., $\mathbf{s}_{m, k}$, times a complex matrix plus the complex conjugate of the TX vector on the $-k$ th carrier, i.e., $\mathbf{s}_{m,-k}^{*}$, times another complex matrix. The latter component is called mirror signal. Also, there is a leakage from the RX noise at the mirror carrier, although its effect is minor. For perfect TX matching $\mathbf{G}_{1}=\mathbf{I}$ and $\mathbf{G}_{2}=\mathbf{0}$ and for perfect RX matching $\mathbf{K}_{1}=\mathbf{I}$ and $\mathbf{K}_{2}=\mathbf{0}$. Hence, for perfect matching in both TX and RX, (8) reduces to (1).

\section{B. Error term}

Subsequently, we regard the influence of IQ imbalance on the estimated TX vector after ZF processing, where we will assume perfect channel knowledge at the RX. Hence, the channel estimate equals the transfer from the TX baseband to the RX baseband and is given by $\hat{\mathbf{H}}_{k}=\mathbf{K}_{1} \mathbf{H}_{k} \mathbf{G}_{1}+\mathbf{K}_{2} \mathbf{H}_{-k}^{*} \mathbf{G}_{2}$, where it is noted that the channel estimate now includes the TX and RX IQ imbalance. When this transfer is used for MIMO processing, the estimated TX signal vector is given by (2).

When studying the influence of TX and RX IQ imbalance, separately, we find that for a system only experiencing TX or RX IQ imbalance, the error term $\varepsilon_{m, k}$ is given by

$$
\begin{aligned}
& \varepsilon_{\mathrm{t}, m, k}=\mathbf{G}_{\mathrm{e}} \mathbf{s}_{m,-k}^{*}+\left(\mathbf{H}_{k} \mathbf{G}_{1}\right)^{\dagger} \mathbf{n}_{m, k}, \\
& \varepsilon_{\mathrm{r}, m, k}=\mathbf{H}_{k}^{\dagger}\left(\mathbf{K}_{\mathrm{e}} \mathbf{H}_{-k}^{*} \mathbf{s}_{m,-k}^{*}+\mathbf{K}_{\mathrm{e}} \mathbf{n}_{m,-k}^{*}+\mathbf{n}_{m, k}\right),
\end{aligned}
$$

respectively. Here $\mathbf{G}_{\mathrm{e}}$ and $\mathbf{K}_{\mathrm{e}}$ are diagonal matrices, defined as $\mathbf{G}_{\mathrm{e}}=\mathbf{G}_{1}^{-1} \mathbf{G}_{2}^{*}$ and $\mathbf{K}_{\mathrm{e}}=\mathbf{K}_{1}^{-1} \mathbf{K}_{2}$.

When comparing the error term due to TX IQ imbalance in (9) to that of RX IQ imbalance in (10), we conclude that the influence of noise in the error term is almost equal. The term $\varepsilon_{\mathrm{r}, m, k}$ also exhibits a noise contribution from the mirror, which is generally minor since the modulus of the elements of $\mathbf{K}_{\mathrm{e}}$ are typically small. Considering the influence of the mirror leakage we can see that the term $\mathbf{G}_{\mathrm{e}} \mathbf{s}_{m,-k}^{*}$ in $\varepsilon_{\mathrm{t}, m, k}$ will be of finite size for given $\mathbf{G}_{\mathrm{e}}$, but that $\mathbf{H}_{k}^{\dagger} \mathbf{K}_{\mathrm{e}} \mathbf{H}_{-k}^{*} \mathbf{s}_{m,-k}^{*}$ in $\varepsilon_{\mathrm{r}, m, k}$ can become infinitely large for given $\mathbf{K}_{\mathrm{e}}$, since it contains the multiplication of the channel matrix for carrier $-k$ with the inverse channel matrix for carrier $k$. When, through fading, the elements of $\mathbf{H}_{-k}$ are large and the elements in $\mathbf{H}_{k}$ are small, the error term will become large. Since errors in detection are caused by the tails of the error distribution, we can conclude qualitatively that in faded channels the influence of RX IQ imbalance will be larger than that of a comparable TX IQ imbalance.

\section{Impact on system performance}

Although the above provides a qualitative distinction between the influence of TX and RX IQ imbalance, a quantitative study was required to provide a more precise understanding of the impact on system performance. For that purpose we derived closed-form expressions for the symbol-error rate (SER) for $M$-QAM symbols in a MIMO OFDM system impaired by IQ mismatch in [13].

From these results one can conclude the following. For TX IQ imbalance, the leakage consists of scaled and rotated $M$-QAM symbols, see (9). In detection this can be considered equal to shifting of the decision boundaries. Consequently, the 
SNR vs. SER curves shift, but do not yield a performance floor for typical values of IQ imbalance. Flooring does, however, occur for extreme values of IQ imbalance, when the decision boundaries are shifted such that the noiseless constellation points are located in the incorrect decision region.

For the RX IQ imbalance, the mirror leakage consists of $M$-QAM symbols multiplied with the channel matrix, see (10). For a Rayleigh-faded channel, the leakage can be approximated as an additional Gaussian RX noise term. As such, the influence is similar to that of the commonly studied AWGN source. For low SNR values the SER curve will follow the non-impaired SER curve and the IQ imbalance causes a SER floor for high SNRs. As for conventional RX noise impaired MIMO systems, the SER performance (and thus the SER flooring) depends on the MIMO configuration.

\section{NONLINEARITIES}

Several elements in analog front-ends, such as amplifiers, $\mathrm{A} / \mathrm{D}$ and $\mathrm{D} / \mathrm{A}$ converters and mixers, are inherently nonlinear. These nonlinearities (NLs) have been neglected for traditional wireless systems, but for OFDM systems the high signal dynamics make the consideration of these effects in the design and evaluation of wireless systems important. Therefore this section reviews the impact of NLs on system performance.

\section{A. System model}

When deriving the influence of NLs on the signal model, we can use the findings of Bussgang [14], since the OFDM signal very much resembles a complex Gaussian signal. Consequently, the signal output of a NL can be written as a scaled (and rotated) version of the input signal plus some statistically independent distortion process. When we again separately study the TX and RX impairments, the received signal vectors are, consequently, given by [4, Chapter 6]

$$
\begin{aligned}
& \mathbf{x}_{\mathrm{t}, m, k}=\mathbf{H}_{k} \boldsymbol{\alpha}_{\mathrm{t}} \mathbf{s}_{m, k}+\mathbf{H}_{k} \mathbf{e}_{\mathrm{t}, m, k}+\mathbf{n}_{m, k}, \\
& \mathbf{x}_{\mathbf{r}, m, k}=\boldsymbol{\alpha}_{\mathrm{r}} \mathbf{H}_{k} \mathbf{s}_{m, k}+\mathbf{e}_{\mathrm{r}, m, k}+\mathbf{n}_{m, k},
\end{aligned}
$$

where the $N_{\mathrm{x}}$-dimensional matrix $\boldsymbol{\alpha}_{\mathrm{x}}$ models the scaling for AM-AM NLs, and phase rotation for AM-PM NLs, and is given by $\operatorname{diag}\left\{\alpha_{\mathrm{x}, 1}, \ldots, \alpha_{\mathrm{x}, N_{\mathrm{x}}}\right\}$ for $\mathrm{x} \in\{\mathrm{t}, \mathrm{r}\}$. For NLs with memory, $\boldsymbol{\alpha}_{\mathrm{x}}$ is a function of $k$. The distortion term is contained in the $N_{\mathrm{x}} \times 1$ vector $\mathbf{e}_{\mathrm{x}, m, k}$. The elements of $\mathbf{e}_{\mathrm{t}, m, k}$ and $\mathbf{e}_{\mathrm{r}, m, k}$ can be shown to be approximately Gaussian distributed with a variance of $\sigma_{\mathrm{e}}^{2}$, especially when clipping of the input signals occurs. Here $\sigma_{\mathrm{e}}^{2}$ and $\alpha_{\mathrm{x}, n}$ are dependent on the experienced NLs and the dynamics of the signals input to the NLs.

\section{B. Error term}

When ZF processing with perfect channel knowledge is applied to the received signal $\mathbf{x}_{m}$ in (11) and (12), the result is given by (2). The error terms are then given by

$$
\begin{aligned}
& \boldsymbol{\varepsilon}_{\mathrm{t}, m, k}=\left(\mathbf{I}-\boldsymbol{\alpha}_{\mathrm{t}}\right) \mathbf{s}_{m, k}+\mathbf{e}_{\mathrm{t}, m, k}+\mathbf{H}_{k}^{\dagger} \mathbf{n}_{m, k}, \\
& \boldsymbol{\varepsilon}_{\mathrm{r}, m, k}=\left(\mathbf{I}-\boldsymbol{\alpha}_{\mathrm{r}}\right) \mathbf{s}_{m, k}+\mathbf{H}_{k}^{\dagger}\left(\mathbf{e}_{\mathrm{r}, m, k}+\mathbf{n}_{m, k}\right),
\end{aligned}
$$

respectively. For (14) the assumption was made that $\alpha$ is branch independent, i.e., $\boldsymbol{\alpha}_{\mathrm{r}}=\alpha_{\mathrm{r}} \mathbf{I}_{N_{\mathrm{r}}}$.

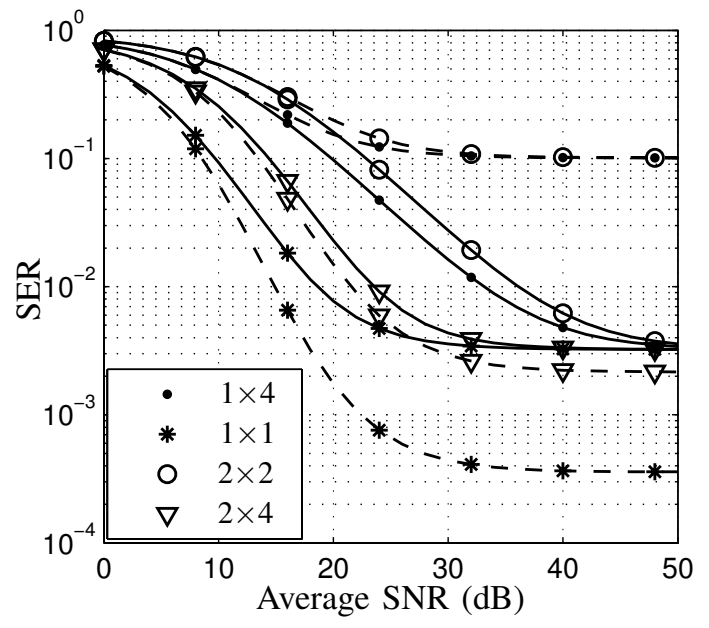

Fig. 3. Comparison of the analytical SER results for TX nonlinearities (solid lines) and RX nonlinearities (dashed lines) for different 16-QAM MIMO systems, $N_{\mathrm{c}}=1024$ and clipping amplifiers with an input backoff of $3 \mathrm{~dB}$.

We can observe from (13) and (14) that the error term for both TX and RX NLs consists of both a signal dependent and conventional RX noise induced error term. For TX NLs we additionally observe a TX distortion noise term and for RX NLs an additional RX distortion noise term.

\section{Impact on system performance}

The impact of the error terms in (13) and (14) on the SER was derived analytically in [15] for MIMO OFDM systems in Rayleigh-faded channels. It can be concluded from these results that, for high SNR values, the TX NLs cause performance floors, which are independent of the MIMO configuration. For RX NLs, in contrast, the performance in this high SNR region does depend on the MIMO configuration. This is illustrated by the numerical SER results in Fig. 3.

The observed differences between the performance impact of TX and RX NLs in MIMO OFDM systems can intuitively be understood as follows. The influence of the signal dependent error term can be seen as a shifting of the decision boundaries, which is similar for the TX and RX NLs. The additive distortion noise term, however, effectively creates an additional TX or RX noise source for the TX and RX NLs, respectively. The effects of this on the SER are similar as for the ICI-related error term in Section III-C.

\section{A GENERALIZED ERROR MODEL}

In the previous sections the influence of the different RF front-end impairments in MIMO OFDM systems were reviewed independently. A typical system would, however, experience a combined influence of these non-idealities. Hence, it is useful to study their combined influence. Although the nature of the different imperfections is rather different, their influence on the detected symbols has a high level of similarity. All treated imperfections in this paper result in a multiplicative and an additive error term. Moreover, we observed a distinct difference between the impact of TX and RX imperfections.

The standard yardstick to measure the aggregate severeness of transceiver imperfections is the error vector magnitude 
(EVM), see e.g. [16]. The EVM is widely used in communication system characterization equipment and specified in standards. It basically measures the root mean square error in the estimated symbols. When the disturbances are distributed with a given (zero-mean) distribution and are independent of the data signal, this directly maps to the system performance, such as symbol- or packet-error rate. It was shown in the present paper, however, that when the impairments induced by the analog front-end of the system prevail, these assumption will not be valid, not even approximately, disqualifying the EVM as an appropriate performance measure.

Therefore an extended EVM (EEVM) model for the combined influence of RF front-end impairments has been developed in [17] and [4, Chapter 7], which includes a multiplicative and an additive error term and does make a distinction between TX and RX imperfections. The following will briefly review the model. The influence of the error model on the $k$ th carrier of the $m$ th symbol of signal vector a is modeled as

$$
\tilde{\mathbf{a}}_{m, k}=\boldsymbol{\chi}_{\mathbf{x}, m, k} \mathbf{a}_{m, k}+\boldsymbol{\eta}_{\mathbf{x}, m, k}
$$

where $\mathrm{x} \in\{\mathrm{t}, \mathrm{r}\}$ for the TX and RX model, respectively. Here $\chi_{\mathbf{x}, m, k}$ is a diagonal matrix modeling the multiplicative error, i.e., scaling and a phase shift, and the vector $\boldsymbol{\eta}_{\mathrm{x}, m, k}$ models the additive error vector with zero-mean entries. The error model is implemented at both sides of the communication link. Then, using (15) and (1), we can define the RX signal vector as

$$
\mathbf{x}_{m}=\chi_{\mathrm{r}, m} \mathbf{H}\left(\boldsymbol{\chi}_{\mathrm{t}, m} \mathbf{s}+\boldsymbol{\eta}_{\mathbf{t}, m}\right)+\boldsymbol{\eta}_{\mathbf{r}, m}+\mathbf{n}_{m} .
$$

We note that the influence of the regarded impairments can all be modeled as affine linear functions, which means that, by definition, their superposition is again affine linear. Hence, since the influence of the separate impairments can be modeled using the proposed model, also their combined influence can be modeled with the model. This is illustrated here for the superposition of a nonlinearity, PN and IQ imbalance in the RX. For that case $\chi_{\mathrm{t}, m, k}=\mathbf{I}, \boldsymbol{\eta}_{\mathbf{r}, m, k}=\mathbf{0}$ and the multiplicative RX term is given by $\chi_{\mathrm{r}, m, k}=\gamma_{0, m} \mathbf{K}_{1} \boldsymbol{\alpha}_{\mathrm{r}}$. The additive RX error term by

$$
\begin{aligned}
& \boldsymbol{\eta}_{\mathbf{r}, m, k}=\mathbf{K}_{1}\left(\gamma_{0, m} \mathbf{e}_{m, k}+\boldsymbol{\xi}_{m, k}\right) \\
& \quad+\mathbf{K}_{2}\left(\gamma_{0, m}^{*}\left\{\boldsymbol{\alpha}_{\mathrm{r}}^{*} \mathbf{H}_{-k}^{*} \mathbf{s}_{m,-k}^{*}+\mathbf{e}_{m,-k}^{*}\right\}+\boldsymbol{\xi}_{m,-k}^{*}\right) .
\end{aligned}
$$

For the mapping of the proposed error model to MIMO OFDM system performance, the reader is referred to [4, Chapter 7].

\section{CONCLUSIONS AND DisCUSSION}

The application of digital signal processing methods for the suppression of the influence of RF front-end imperfections is a very promising path to meet the challenging requirements for next-generation high-rate wireless communication systems. For this to be successful, a thorough understanding of the influence of the different RF impairments on system performance is crucial. Next to the generally considered RX impairments, also TX impairments will become important when measures enabling compensation are included in standards for communication systems, like what is now already common practice for carrier frequency offsets.
Therefore this paper reviewed the impact of phase noise, IQ imbalance and nonlinearities in multiple-antenna OFDM systems and revealed the difference between TX and RX imperfections in fading environments. It was shown that the relative impact of TX and RX non-idealities is highly dependent on the MIMO configuration, i.e., the number TX and $\mathrm{RX}$ branches. Moreover, a generalized error model, named extended EVM (EEVM), was reviewed which attempts to capture the combined influence of the different RF impairments in a convenient affine linear model. This model is more appropriate than EVM for system where RF imperfections prevail.

Hence, the TX/RX-asymmetry design problem for a given MIMO configuration can be considered in the following way. A cost-limited optimization of the system performance based on the EEVM model to come to an allowable level of $\mathrm{TX} / \mathrm{RX}$ impairments in RF front-end production, which can be suppressed sufficiently with fixed processing capabilities in TX (predistortion) and/or RX (compensation).

\section{REFERENCES}

[1] G. Fettweis, M. Löhning, D. Petrovic, M. Windisch, P. Zillmann, and W. Rave, "Dirty RF: A new paradigm," in Proc. PIMRC 2005, Berlin, Germany, Sept. 2005, vol. 4, pp. 2347-2355.

[2] T. C. W. Schenk, RF Impairments in Multiple Antenna OFDM: influence and mitigation, Ph.D. thesis, Eindhoven Univ. of Technology, Nov. 2006.

[3] T. C. W. Schenk, X.-J. Tao, P. F. M. Smulders, and E. R. Fledderus, "On the influence of phase noise induced ICI in MIMO OFDM systems," IEEE Commun. Letters, vol. 9, pp. 682-684, Aug. 2005.

[4] T. C. W. Schenk, RF Imperfections in High-rate Wireless Systems: Impact and Digital Compensation, Springer, 2008.

[5] H. Steendam, The Effect of Synchronisation Errors on Multicarrier Systems, Ph.D. thesis, Universiteit Gent, 2000.

[6] P. Robertson and S. Kaiser, "Analysis of the effects of phase-noise in orthogonal frequency division multiplex (OFDM) systems," in Proc. ICC'95, 1995, vol. 3, pp. 1652-1657.

[7] L. Tomba, "Analysis of phase noise effects in OFDM modems," IEEE Trans. on Commun., vol. 46, no. 5, pp. 580-583, May 1998.

[8] A. G. Armada, "Understanding the effects of phase noise in orthogonal frequency division multiplexing (OFDM)," IEEE Trans. on Broadcasting, vol. 47, no. 2, pp. 153-159, June 2001.

[9] L. Piazzo and P. Mandarini, "Analysis of phase noise effects in OFDM modems," IEEE Trans. on Commun., vol. 50, pp. 1696-1705, Oct. 2002.

[10] D. Petrovic, W. Rave, and G. Fettweis, "Properties of the intercarrier interference due to phase noise in OFDM," in Proc. International Conf. on Commun., May 2005, vol. 4, pp. 2605-2610.

[11] T. C. W. Schenk, R. W. v. d. Hofstad, E. R. Fledderus, and P. F. M Smulders, "Distribution of the ICI term in phase noise impaired OFDM systems," IEEE Trans. on Wireless Comm., vol. 6, pp. 1488-1500, 2007.

[12] A. A. Abidi, "Direct-conversion radio transceivers for digital communications," IEEE Journ. of Solid-State Circuits, vol. 30, pp. 1399-1410, Dec. 1995.

[13] T. C. W. Schenk, E. R. Fledderus, and P. F. M. Smulders, "Performance analysis of zero-IF MIMO OFDM transceivers with IQ imbalance," Journal of Communications, vol. 2, no. 7, pp. 9-19, Dec. 2007.

[14] J. J. Bussgang, "Crosscorrelation functions of amplitude-distorted Gaussian signals," Tech. Rep. 216, Research Lab. Electron, M.I.T., Cambridge, MA, Mar. 1952.

[15] T. C. W. Schenk, P. F. M. Smulders, and E. R. Fledderus, "Impact of nonlinearities in multiple-antenna OFDM transceivers," in Proc. SCVT2006, Liege, Belgium, Nov. 2006, pp. 53-56.

[16] R. Hassun, M. Flaherty, R. Matreci, and M. Taylor, "Effective evaluation of link quality using error vector magnitude techniques," in Proc. 1997 Wireless Commun. Conf., Aug. 1997, pp. 89-94.

[17] T. C. W. Schenk, X.-J. Tao, P. F. M. Smulders, and E. R. Fledderus, "A two-step modeling approach for the influence of radio-system impairments in multicarrier MIMO systems," in Proc. PIMRC 2005, Berlin, Germany, Sept. 2005, pp. 176-180. 